\title{
Quantitative Assessment of the Cryolipolysis Method for Body Contouring in Asian Patients
}

\author{
Ayaka Nishikawa' \\ Yoshiyuki Aikawa $\mathbb{D}^{2}$ \\ 'Cosmetic Dermatology, SBC Medical \\ Group, Medical Corporation Shoubikai, \\ Shinjuku-ku Tokyo, Japan; ${ }^{2}$ Cosmetic \\ Surgery, SBC Medical Group, Shinjuku-ku \\ Tokyo, Japan
}

Purpose: Cryolipolysis has been demonstrated to be a safe and efficient non-invasive treatment modality for reducing subcutaneous discrete areas of fat; however, only limited studies have focused on body contouring in Asian patients. The present work focuses on the quantitative assessment of the cryolipolysis method for body contouring in Asian patients.

Patients and Methods: The retrospective study evaluated the medical records of 4122 patients with body contouring who underwent cryolipolysis treatment in multiple centers across Japan from January 2019 to June 2019. We evaluated the demographic profiles, treatment areas, and safety assessments among these 4122 patients. The effectiveness of the treatment was assessed in 146 patients at one of the clinics, Shonan Beauty Clinic, out of which 54 and 27 had treatments in the abdomen and upper arm areas, respectively. Clinical outcomes were assessed based on patient surveys, circumference measurements, and assessment of photographs.

Results: Evaluation of choice for treatment area suggested that men generally focused more on the surrounding areas of the abdomen, while women even had options for the abdomen and the upper arm. No long-term side effects were observed during the study period. There was a significant reduction in the circumference of both the abdomen and upper arm areas $(\mathrm{P}<0.05)$. Additionally, $16.7 \%$ and $18.5 \%$ of patients who had received treatment on their abdomen and upper arm, respectively, returned for the next sessions within six months. Patients who had received treatment on their upper arms were more satisfied in the categories of pain and bruising and treatment effectiveness compared to those who had received treatment on their abdomen.

Conclusion: Based on the results obtained in this retrospective study on Asian patients, it can be stated that cryolipolysis is a safe and well-tolerated nonsurgical fat-reduction procedure. Clinical effectiveness can be consistently achieved with proper patient evaluation and patient satisfaction.

Keywords: cryolipolysis, body contouring, retrospective study, Asian patients, non-invasive technique, safety testing

\section{Introduction}

Non-invasive body contouring is indeed one of the most appealing areas of esthetic surgery at the present time. Supported by growing consumer demand for better and faster recovery procedures with fewer side effects, novel treatment modalities have been crafted to tackle body contouring from a comparatively less invasive aspect. ${ }^{1}$

Existing surgical procedure measures represent the drawback of hospitalization, use of anesthetics, pain, swelling, involvement of long-term recovery period, as well as the intrinsic risk associated with surgery. ${ }^{2}$ A growing number of customers looking for esthetic body contouring changes are interested in procedures, which 
are less traumatic and more effective than conventional suction assisted liposuction (SAL). Although SAL remains perceptible as a mainstay in body contouring techniques for plastic surgeons, current trends in energy-based body contouring devices, including third-generation ultrasound, laser-assisted lipolysis (LAL), and liposuction assisted by $\mathrm{RF}$, may offer reduced bruising, swelling, discomfort, and increased skin contraction when correlated with SAL. ${ }^{3}$ Yet, as common as the various types of liposuction, the fastest-growing sector in the esthetic medicine sector is in the area of non-invasive body contouring. Various technologies for contouring the body that involve transepidermal energy delivery targeted at adipocytes include suction massage devices, suction massage thermal devices, radiofrequency energy devices, high-frequency focused ultrasound energy devices, cryolipolysis energy devices, and low-level light laser therapy devices. ${ }^{4}$

Body size and patient BMI have grown considerably over the past decade, globally, followed by business development and technical advancements dedicated to nonsurgical management of fat and body contouring. The fundamental research of non-invasive body contouring involves the adipocyte, its triglyceride storage, and the cumulative quantity of adiposities with specific and distributed surplus for adipose tissue, the convex distension defining the focal protrusions, and superficial cellulite topographically. ${ }^{5}$ Adipocyte is an essential cell, which is engaged in energy storage, hormone control, and a multitude of other roles in endocrinology. The adipocyte does have a significant quantity of cytoplasm that is a function of triglyceride storage depot, which consists mainly of glycerol and free fatty acids. Adiposities tend to swell with triglycerides when calorific intake outweighs caloric output. As adiposities proceed to expand within their respective parts, they form protrusions or soft tissue distensions. ${ }^{6}$

Prior clinical studies indicated cryolipolysis could safely and effectively eliminate localized fat for non-invasive body contouring applications. The cryolipolysis methodology involves using a regulated thermal reduction process that leads to selective adipocyte cell death or apoptosis via controlled cooling. Earlier reports suggested the efficacy of the treatment module in porcine models and in humans. ${ }^{7}$ The use of temperatures below average but above freezing causes apoptosis-mediated death in cells. The adiposities are much more responsive than other cells to the cooling process, leading to limited collateral damage to the tissues surrounding them. ${ }^{8}$ The process is conveniently done without anesthetics or pain relievers in a healthcare setting. After applying a gel pad and applicator to the patient's skin, the tissue storing the specific fat layer is held into the evacuated applicator by a control unit. Sensors on the cooling surfaces of the applicator monitor the skin surface, managing feedback that controls the rate of heat flux, sustained 45 and 60 minutes. With limited pain, the patient quickly returns to normal routines following the procedure.

The present work focuses on the quantitative assessment of the cryolipolysis method for body contouring by examining the patient demographics, safety, and effectiveness of cryolipolysis in Asian patients.

\section{Materials and Methods Study Design}

The study retrospectively evaluated medical records of 4122 patients with body contouring in multiple centers (30 clinics), who underwent CoolSculpting ${ }^{\circledR}$ (manufactured by ALLERGEN AESTHETICS, AN ABBVIE COMPANY, IRVINE, CA, USA) from January 2019 to June 2019. We evaluated the demographic profiles, popular treatment areas, and side effects among 4122 patients. The safety of the procedure was analyzed in all the patients receiving the treatment in terms of adverse drug reactions. The study was approved by Medical Corporation Shoubikai Ethics Committee with an approval number-2-009. Informed consent was obtained in the form of an opt-out. The study was conducted in accordance with the Declaration of Helsinki.

Before starting the procedure, patients typically met with a physician for an initial consultation, during which areas of concern were discussed, realistic expectations for fat reduction were established, and the sites for applicator placement and the number of cycles were planned based on a whole-body assessment. The treatment areas were prioritized to develop a plan that would satisfy the patients' needs and budgetary restrictions.

\section{Evaluation of Treatment Effectiveness}

Within a period of 6 months, the cryolipolysis treatment effectiveness among customers was evaluated at one of the clinics, Shonan Beauty Clinic, in 146 patients. Since the effectiveness of CoolSculpting ${ }^{\circledR}$ could not be properly evaluated in cases of overall body weight changes, cases with $>5 \%$ body weight change were excluded from the study. Clinical outcomes of treatment were examined based on circumference measurements and assessment of digital 
photographs and the satisfaction of patients based on the survey. The abdomen and upper arms were selected as the targeted area for treatment because the most popular part is the abdomen, and the next part is upper arms among 4122 patients. Of these patients, 54 and 27 had treatments in the abdomen and upper arm areas, respectively. Additionally, 14 patients had treatment in the lower back, 8 in the back, 12 in the buttocks, and 31 in the thighs. The effectiveness of the treatment was evaluated at baseline and after the treatment procedure.

\section{Statistical Analysis}

Descriptive statistics were used to report the number and percentage of categorical variables using Microsoft Excel. A paired $t$-test was performed to compare the differences in circumference in the abdomen and upper arm before and after the treatment. A p-value $<0.05$ was considered to be statistically significant.

\section{Results}

A total number of 4122 patients were included in the study. Out of the total patients enrolled for the study, $8.6 \%$ were males, and $91.4 \%$ were females (Figure 1) The average age of the patients was 34.7 years old. The age distribution of the patients is represented in (Figure 2). Of these patients, $97.55 \%$ were Japanese, $1.67 \%$ were Chinese, while $0.78 \%$ of the patient population were

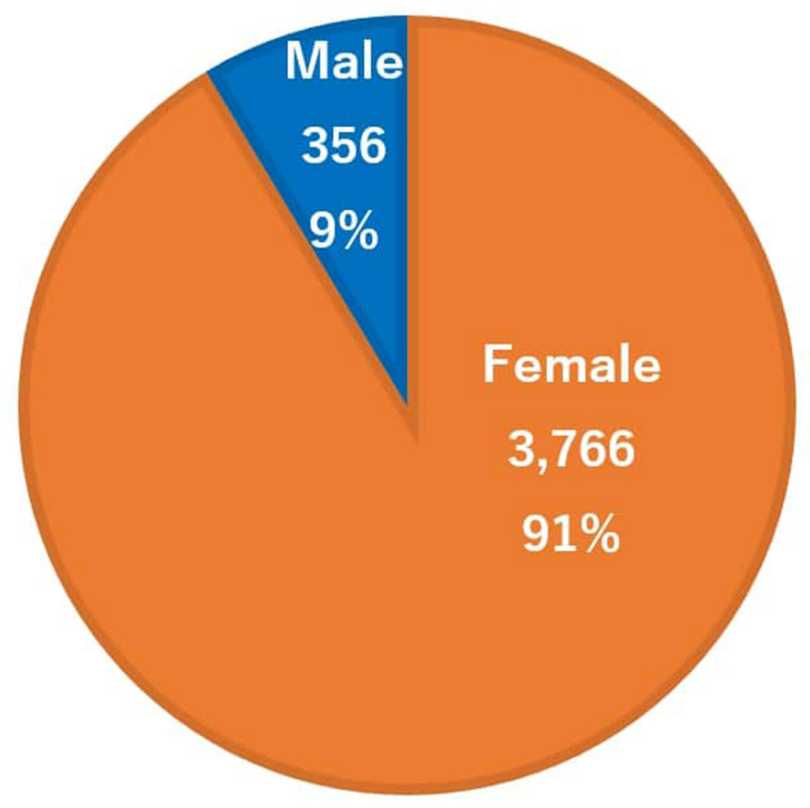

Figure I Sex distribution of the patients. The sex distribution of patients in this series was $91.4 \%$ female, and $8.6 \%$ male.

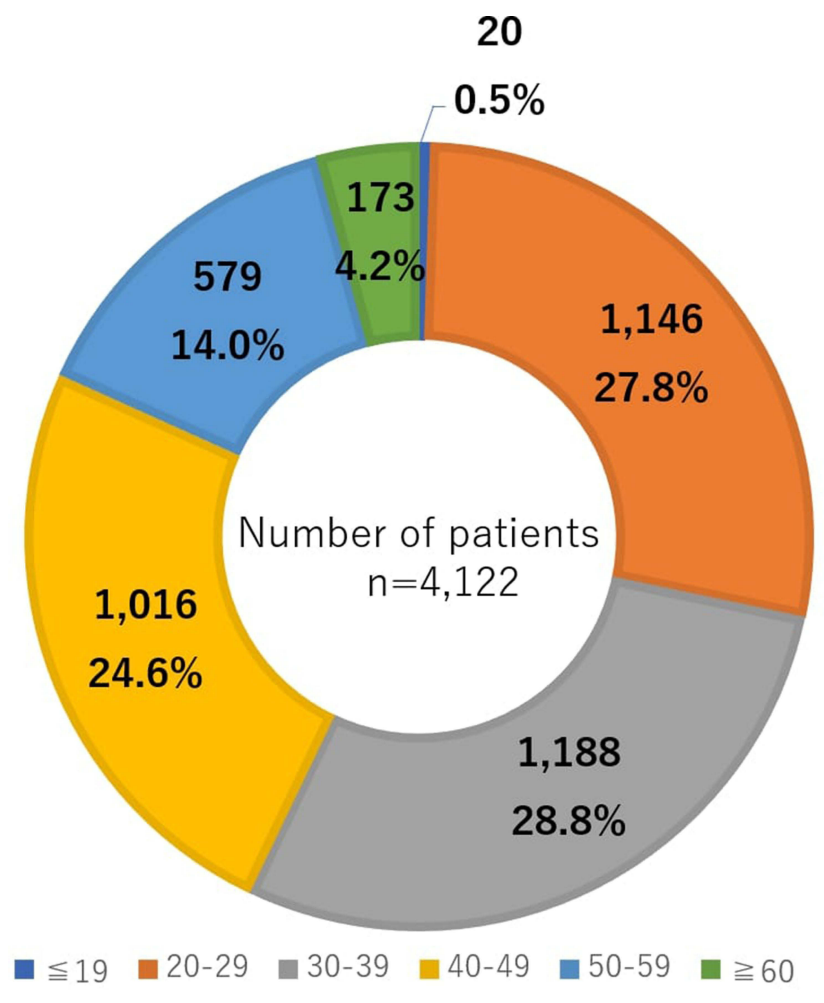

Figure 2 Age distribution of the patients. The age distribution of patients in this series was $30 \% 30 \pm 39$ years, $29 \% 20 \pm 29$ years, $24 \% 40 \pm 49$ years, $14 \% 50 \pm 59$ years, and $3 \% \pm 19$ years. The average age was 34.7 years.

from other groups (Figure 3). There were $46.3 \%$ of the patients who underwent CoolSculpting ${ }^{\circledR}$ were, in fact, first-time visitors in the clinic. The evaluation of choice for treatment area among these cases demonstrated that men generally focused more on the abdomen's surrounding areas. At the same time, women had rather even choices for the treatment areas (Figure 4). The rates of applicator use at various sites are shown in (Figure 5).

\section{Safety Assessment}

Regarding the 4122 patients who were assessed for the safety of cryolipolysis, no reports of persistent erythema, frostbite, paradoxical lipohypertrophy, or skin necrosis were noted in the patients with no long-term side effects were observed. However, 6 associated complications (hyper-pigmentation, $\mathrm{n}=3$; frostbite, $\mathrm{n}=3$ ) were reported in the treatment areas post the study period. Hyperpigmentation was reported after 10-62 days after treatment, and frostbite was reported 4-8 days after treatment.

\section{Clinical Efficacy}

Of the total 146 patients, 54 and 27 patients had treatments in the abdomen and upper arm areas. Out of patients who 


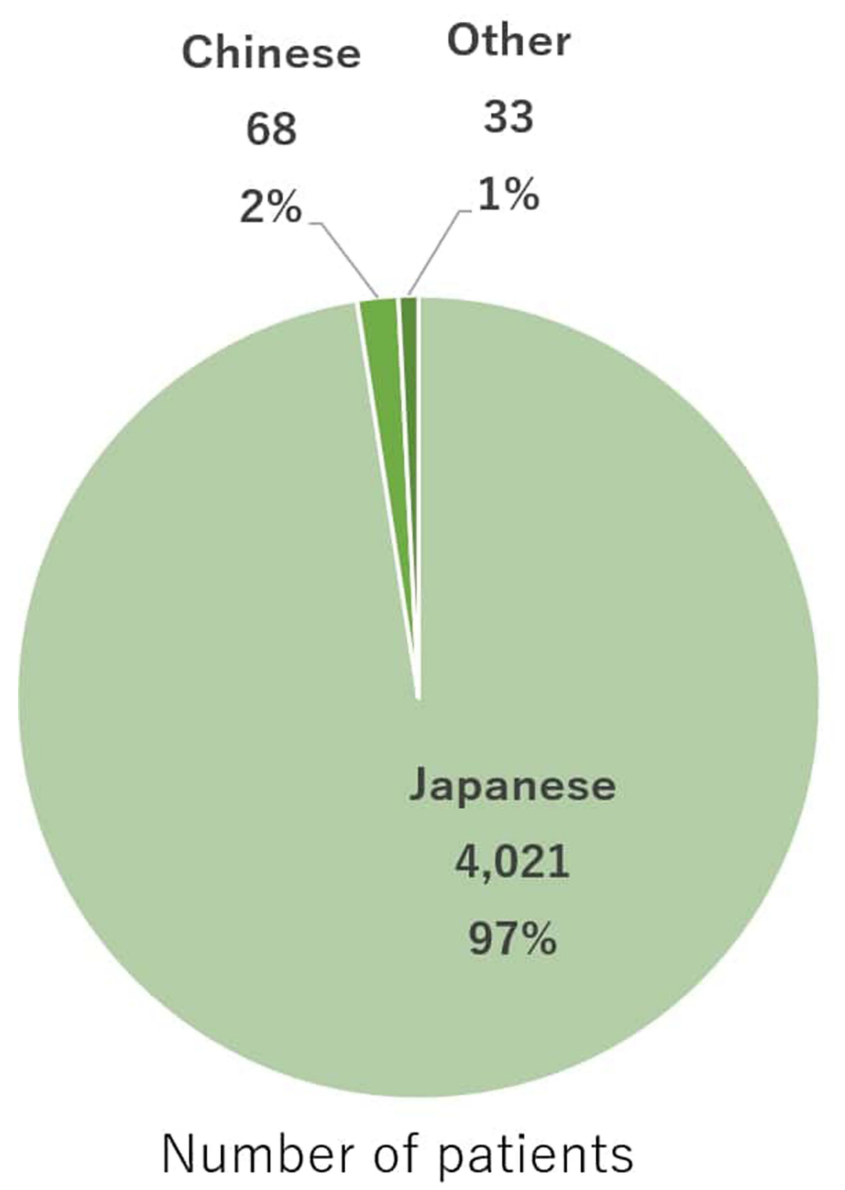

$\mathrm{n}=4,122$

Figure 3 Ethnic distribution of the patients. The ethnic distribution of patients in this series was $97.55 \%$ Japanese, $1.67 \%$ Chinese, and $0.78 \%$ other.

had treatment in the abdomen, 9.3\% were males, and $90.7 \%$ were females, and of those who had treatment in the upper arm, 3.7\% were males, and $96.3 \%$ were females. The circumference of the abdomen was measured at $10 \mathrm{~cm}, 5 \mathrm{~cm}$, above and below the navel, and around the navel level. The sum of these numbers was then compared at baseline and after the treatment procedure. Similarly, the circumference of the upper arm was measured at $15 \mathrm{~cm}$, $10 \mathrm{~cm}, 5 \mathrm{~cm}$ above the elbow, and the sum of these numbers was then compared before/after the treatment procedure.

For the abdomen evaluation, the circumference was measured at 5 different positions that were further summed up. An average circumference decrease of $2.7 \mathrm{~cm}(P<0.05)$ in total was noted in each treatment session. In the upper arm, an average reduction of $1.3 \mathrm{~cm}(P<0.05)$ was observed (Table 1).

\section{Patient Satisfaction Score}

Patients' satisfaction survey included only those who received treatment on their abdomen $(n=54)$ and the upper $\operatorname{arm}(\mathrm{n}=27)$.

The patients were asked for feedback on three aspects: (1) pain, swelling, or bruising during and after the treatment, (2) the results of the treatment, and (3) whether the patient felt happier after the treatment. The responses were scored on a 5-point grade scale. These questionnaire results are presented in (Table 2).

It was noted that $16.7 \%$ of patients who had received treatment on their abdomen returned for the next sessions in the same area within 6 months. Similarly, $18.5 \%$ of patients who had received treatment on their upper arms also returned for more treatment sessions.

Patients who had received treatment on their upper arms were more satisfied in the categories of pain and bruising and treatment effectiveness compared to those who had received treatment on their abdomen.

Following the treatment session, bruising was observed in some patients after the procedure (Figure 6). The treatment effectiveness of patients following one session and two treatment procedures is presented in (Figures 7 and 8), respectively.

\section{Discussion}

Over the last several years, research has advocated using non-invasive techniques of fat reduction as a substitute rather than an adjunct to liposuction, which is identified for elevated risk of associated side effects and complications. To produce a quantifiable outcome, all nonsurgical techniques for body sculpting rely on the concept of either stimulating fat cell necrosis or apoptosis. Diode laser, cryolipolysis, RF, and HIFU are the most popular and widely used non-invasive fat reduction techniques. In a systematic review, their noninvasive studies that performed treatments on the abdomen (with or without flanks) or waist were reported circumferential reductions greater than $2 \mathrm{~cm}$. Furthermore, the only study of cryolipolysis to measure abdominal circumference showed a $6.86 \mathrm{~cm}$ reduction. In addition, studies of cryolipolysis have reported result satisfaction rates of $73-100 \%{ }^{9}$ Cryolipolysis can thus be the first-line treatment choice for non-invasive fat reduction with the maximum efficacy and satisfaction rate. Cryolipolysis is reported to be safe for all skin forms, and for repeated usage. ${ }^{10}$ Potential candidates for significant outcomes following the cryolipolysis procedure are those who indulge in daily exercise, follow a balanced 


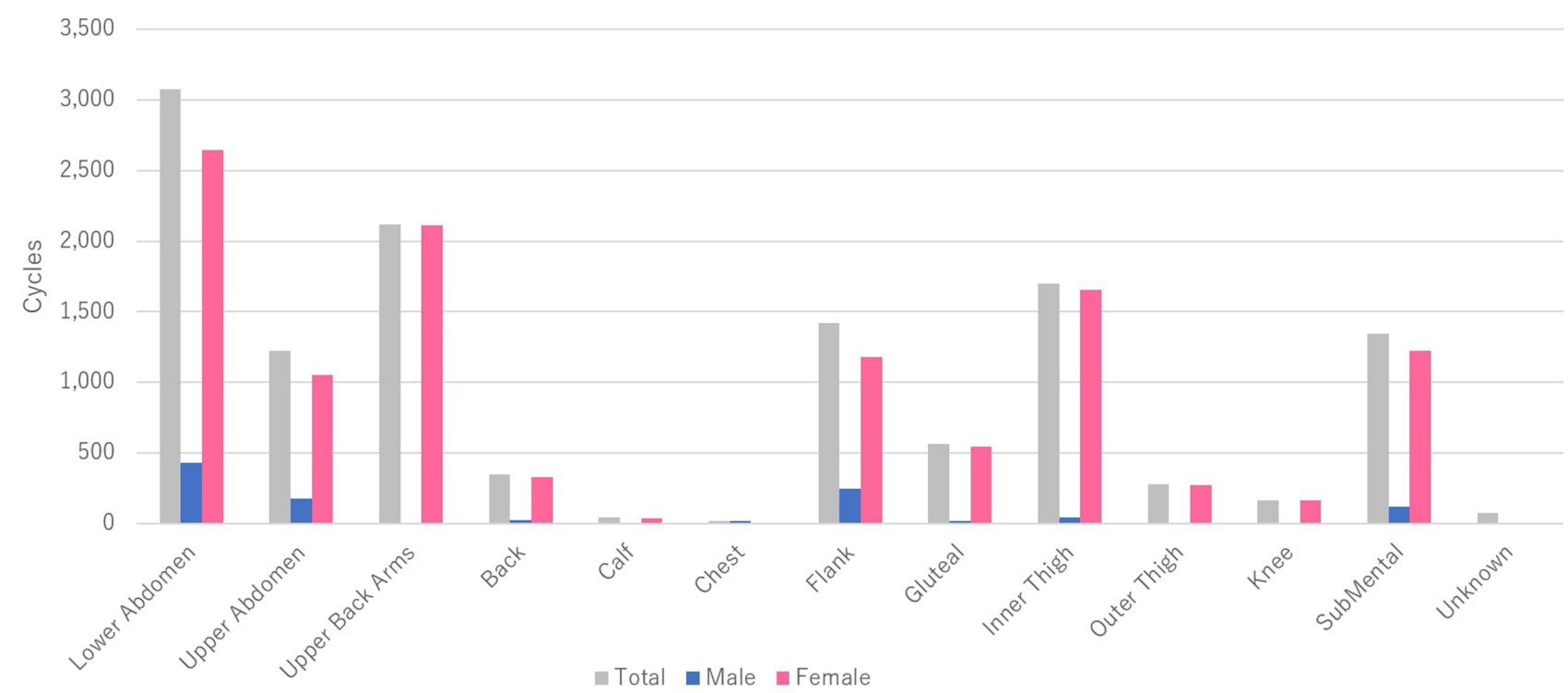

Figure 4 Number of treatment cycles by anatomic site. Total treatments were delivered to the lower abdomen (24.9\%), upper arms (I $7.1 \%)$, inner thigh (I3.7\%), and flank (I I.5\%).

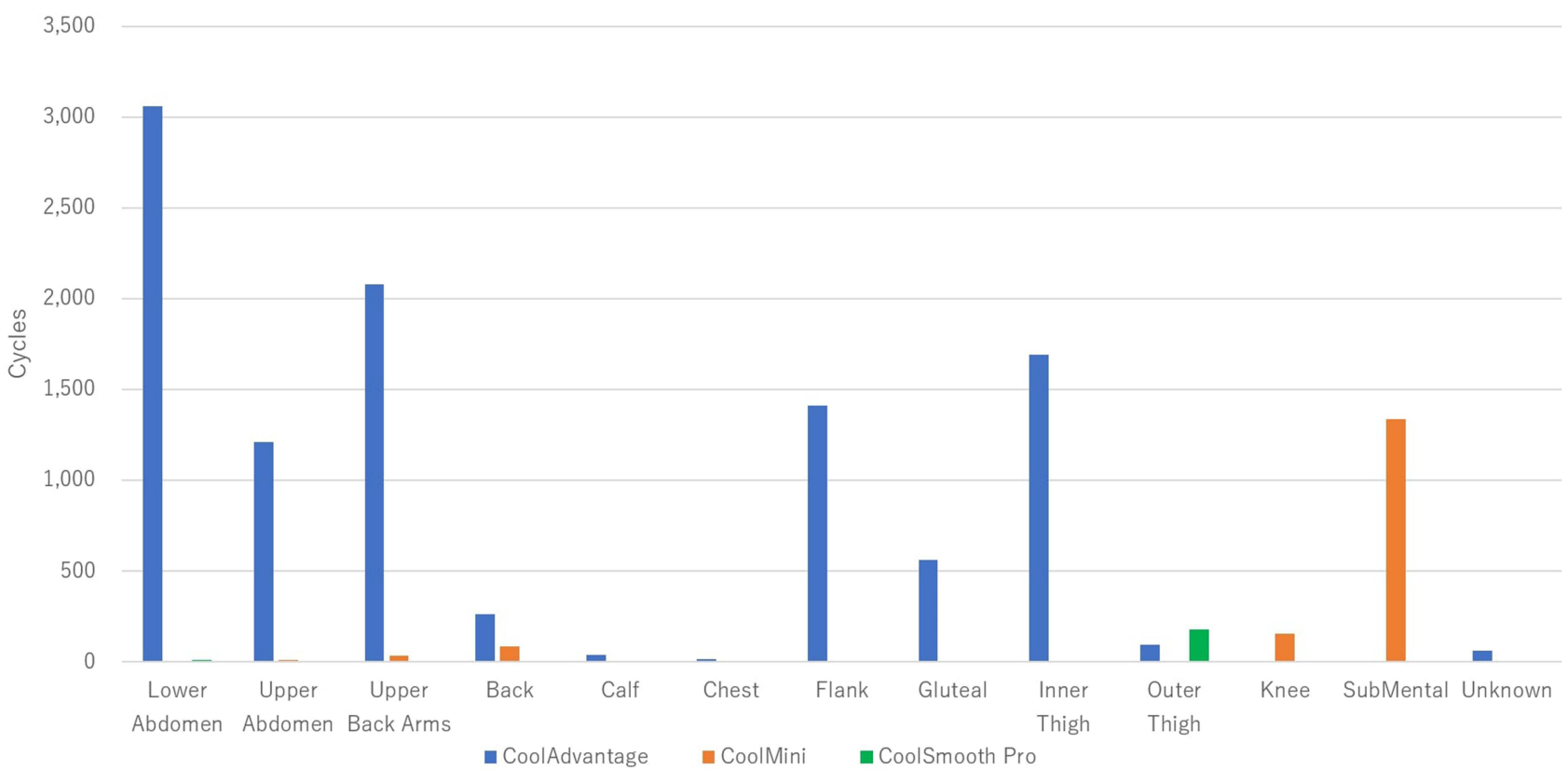

Figure 5 Applicator use per site. CoolAdvantage used in $85 \%$, CoolMini in 13\%, and CoolSmooth Pro in $2 \%$ of all cycles.

diet, have visible fat protrusions in the trunk, are reasonable in their aspirations, and are likely to continue a healthy, active lifestyle. Given that people in Japan are generally hesitant to go to an aesthetic clinic; however, body contouring treatment with a relatively high ratio of new customers could be a gateway for new customers to an esthetic clinic.

Table I Changes in Average Circumference in Total of 5 Measured Positions in Abdomen and Upper Arm

\begin{tabular}{|l|c|c|c|c|}
\hline & Baseline (A) & After Treatment (B) & Circumference Reduction (B-A) & P value \\
\hline Abdomen & $426.4 \pm 52.7$ & $423.7 \pm 52.7$ & $-2.7 \pm 8.2$ & $<0.05$ \\
Upper arms & $174.7 \pm 17.8$ & $173.4 \pm 18.2$ & $-1.3 \pm 4.0$ & $<0.05$ \\
\hline
\end{tabular}

Note: Average score $(\mathrm{cm}) \pm$ S.D.P value is calculated by paired $t$-test. 
Table 2 Questionnaire Results from Those Who Underwent a Treatment on the Abdomen/Upper Arms

\begin{tabular}{|l|c|c|}
\hline & Abdomen & Upper Arms \\
\hline Whether there was any pain, swelling, or bruising during and after the treatment & 3.8 & 4.3 \\
The results of the treatment & 3.4 & 4.0 \\
Whether the patient felt happier after the treatment & 3.3 & 3.7 \\
\hline
\end{tabular}

Note: The table shows average scores. It consists of 5-point scales from I to 5 . Each score represents I=very dissatisfied, $2=$ dissatisfied, $3=$ neutral, $4=$ satisfied, and $5=$ very satisfied.

As far as the safety profile is concerned, many studies have not reported to report any major adverse effects due to cryolipolysis, scarring, ulceration, or disfiguring. Although the application of cold temperatures is widely acknowledged to stimulate cases of subcutaneous panniculitis, no cases of nodulation have been reported to date. ${ }^{8}$ Temporary erythema, bruising, and transient numbness are predicted side effects that typically mitigate within 14 days

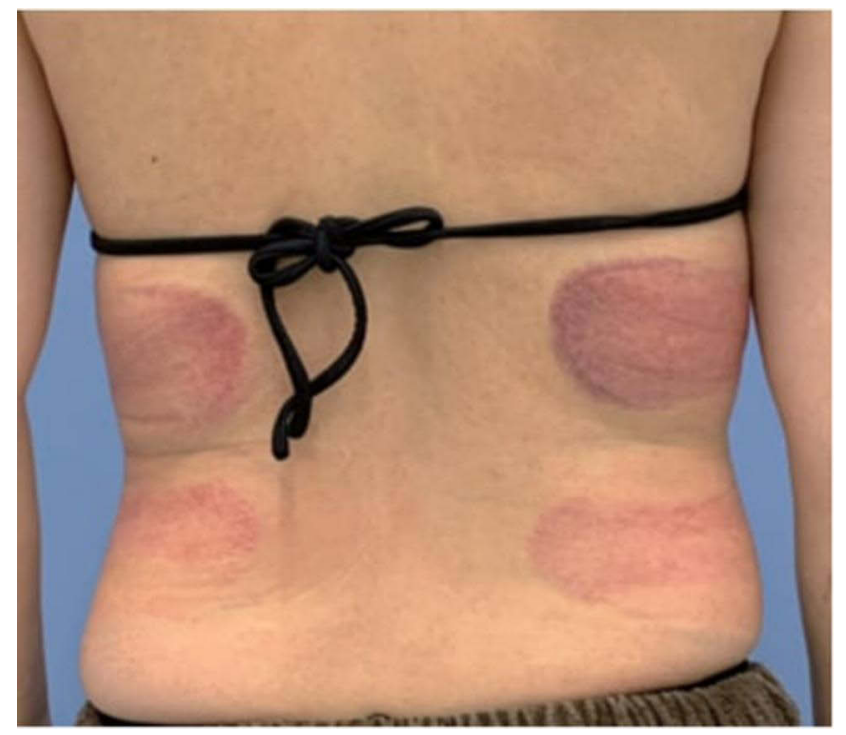

Figure 6 Bruising after the treatment procedure on flanks.
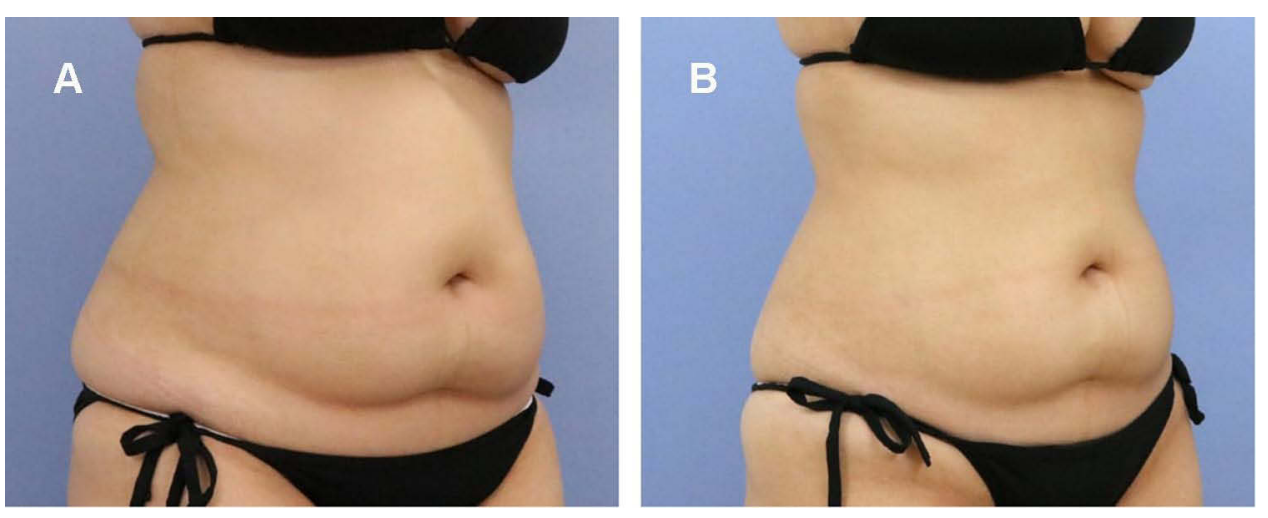

Figure 7 (A) Baseline; (B) 3 months after abdominal treatment with a circumference change above navel of $-6 \mathrm{~cm}$.

of the treatment procedure. ${ }^{11}$ It was observed that six patients out of nine enrolled patients had a temporary decreased sensation that reverted after a period of 3.6 weeks. ${ }^{7}$ However, no alteration in neural function was observed, as evidenced by the biopsy reports. Further, in another study, no significant changes in liver function were noted in a total of 40 patients enrolled for the study after a period of 12 weeks after the cryolipolysis procedure. ${ }^{12}$

In our study, 3 frostbite and 3 hyperpigmentation in the treatment areas were reported. Another study on the safety of cryolipolysis treatment, similar kinds of results were reported in which 16 out of 46 participants had side effects, including pain, erythema, burning/blistering, edema, purpura, crusting, and temporary hyper pigmentation. ${ }^{13}$ In addition, in 4 weeks, all these adverse events were stabilized, and none led to severe complications. The strong safety pattern of cryolipolysis is one of the advantages in comparison to invasive procedures. Serious and lasting side effects due to intense cold stress, such as the development of scarring, ulceration, weakness, and development of subcutaneous panniculitis nodules, had not been recorded elsewhere, which may be assumed as confirmatory support regarding the safety of the procedure. ${ }^{14}$

The results showed the clinical effectiveness of the methodology followed by the enrolled patients. The 

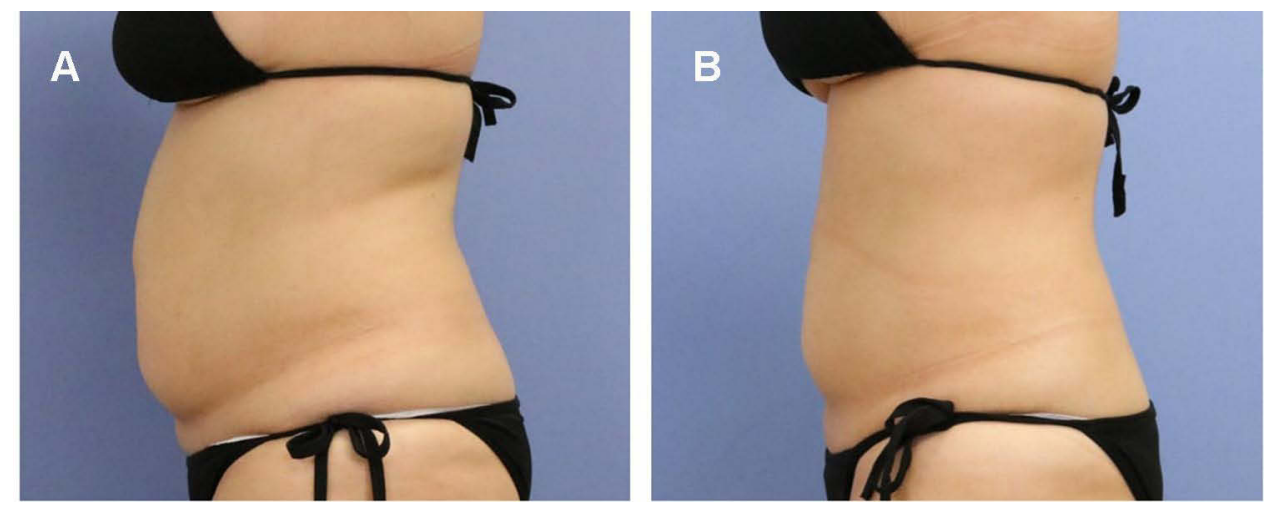

Figure 8 (A) Baseline; (B) I month after 2 abdominal treatments. It visibly shows the reduction on lower abdomen and circumference reduction above navel of - I0.7 cm.

clinical efficacy of the said procedure was conferred by the average circumference reduction of $2.7 \mathrm{~cm}$ and $1.3 \mathrm{~cm}$ in the abdomen and upper arm, respectively, in the study period of 6 months. Accurate and consistent uses of cold temperatures may have induced the adipocyte cell death, which is eventually devoured and digested by macrophages. ${ }^{15}$ Studies support the fact that no changes in subcutaneous fat are immediately apparent following treatment. ${ }^{5}$ An inflammatory cycle induced by adipocyte apoptosis, expressed by an infiltration of inflammatory cells, is observed within three days of treatment, which rises 14 days later as the adiposities become further surrounded by histiocytes, neutrophils, lymphocytes, and other mononuclear cells. Macrophages and other phagocytes populate around, compress, and ingest the lipid cells at 14-30 days after the procedure as part of the body's normal reaction to damage. After four weeks of therapy, the inflammation reduces, and the number of adiposities declines. The interlobular septa become noticeably enlarged, and the inflammatory cycle reduces further two to three months after the treatment procedure. At this stage, the amount of fat in the treated area is evidently reduced, and the separate constitute compensates a majority of the tissue volume. ${ }^{16}$ A retrospective multicenter study recorded that $86 \%$ of the 518 enrolled subjects reported promising results in the abdomen, back, and flank at body sites where cryolipolysis was most successful. ${ }^{17}$ For potential synergistic effects, cryolipolysis was coupled with acoustic waves in an uncontrolled analysis by Ferraro et al. Substantial circumference reductions of up to $6.7 \mathrm{~cm}$ and fat layer thickness of up to $4.5 \mathrm{~cm}$ after 12 weeks were observed after 3-4 treatment cycles. ${ }^{18}$ A good lot of publications have not been reported related to the long-term effect of cryolipolysis. A single study involving two subjects was treated unilaterally on one flank, and then followed for up to 5 years after the said procedure. During this research, the fat reduction was found to be resilient, given changes in body weight during the follow-up period. While little is documented about the longevity of selective cryolipolysis induced fat loss, there are no reported instances where fat loss after cold exposure reappears afterward. ${ }^{8}$

Surprisingly, our patients who had received treatment on their upper arms were more satisfied in the categories of pain and bruising and treatment effectiveness compared to those who had received treatment on their abdomen. The responses of the patients were cumulated in the form of a score on a 5-point grade scale. The observed result was apparent from the patient survey taken after the treatment session. The patients' appreciable response regarding the safety and clinical effectiveness of the treatment methodology in the survey may be due to the significant reduction of localized fat in the treated areas following the treatment procedure. Given the safety and efficacy of such non-invasive body contouring procedures with proven results published, by far, in peerreviewed literature, patient dissatisfaction comes out as the most prevalent associated complication. ${ }^{19,20}$ Patients who present themselves at the esthetic clinics for body contouring treatment often perceive a maximum circumferential reduction in a limited period, as seen with invasive procedures. These kinds of pre-conceptions often lead to dissatisfaction among patients after receiving the treatment. Hence, it is always recommended not to promote such methods over time. The surgeons need to provide detailed knowledge about the nonsurgical procedures' clinical effectiveness in advance. The best choices for the non-invasive procedures are those patients who have acceptance of a mild to a moderate outcome. In reality, the best candidates are the ones who think they 
will be satisfied with any noticeable decreases in localized fat. Hence, it can be stated that proper patient selection before the start of the procedure is a deciding factor for the successful outcome and the acceptance rate among users.

It was noted that $16.7 \%$ of patients who had received treatment on their abdomen returned for the next sessions on the same area within six months, and $18.5 \%$ of patients who had received treatment on their upper arms also returned for more treatment sessions. This result indicated the acceptability of the procedure among a pool of patients.

Our retrospective study demonstrated that $46.3 \%$ of the cryolipolysis patients were new patients. They visited the clinic for the first time, specifically for CoolSculpting ${ }^{\circledR}$, which indicated the popularity of the esthetic patients' said procedure.

Irrespective of the appreciable results, there are certain limitations appended to the present study. Retrospective study design with the involvement of a smaller number of subjects and the single-centric model of the trial for evaluating effectiveness are the major limitations of the study. In addition, detailed mechanistic studies relating to the evaluation of hematological parameters before and after the treatment session would have added value to the current results.

The investigators have found that proper selection of patients, appropriate selection of anatomical locations, and an ample number of treatment cycles are crucial for improving the positive outcome for patients. Additional research needs to be carried out to standardize the treatment time, at many stages to improve the efficiency of cryolipolysis in fibrous protrusions, which are generally hard to treat. Value-added work needs to be carried out, defining the intervention function by which adiposities are impaired by cryolipolysis. It is not established why adiposities are more highly susceptible to cold conditions than other types of cells, and how adipocyte apoptosis progresses and contributes to inflammatory infiltration of the targeted treatment site.

\section{Conclusion}

Cryolipolysis is a new and promising non-invasive method of reducing regional sub-cutaneous fat involving extremely judicious cold-induced adipocyte apoptosis as the core aspect without affecting the nearby tissue. Despite the lack of data in the Asian population, cryolipolysis has illustrated its strong efficacy and safety profile. This may be the first choice of treatment modality for patients who prefer body contouring. Randomized multi-centric clinical studies involving many of the study population with detailed research on the safety aspect of the procedure could add valuable insights about the treatment procedure, which would build-up a considerable customer base in the near future. With demonstrated safety and effectiveness, the prospects of non-invasive body contouring procedures appear vivid. Surgeons who use these techniques will deliver non-invasive and intrusive body contouring and provide concurrent systems designed to transfer patients from invasive to non-invasive ones. A corporate study and marketing recognition behind the business model for patients who desire non-invasive services need to be placed in the emerging area of non-invasive surgical procedures.

\section{Data Sharing Statement}

All the data related to the study are available in the manuscript.

\section{Ethics Approval}

The study was approved by the Medical Corporation Shoubikai Ethics Committee with an approval number2-009.

\section{Consent for Publication}

All the authors agreed for the publication of this manuscript.

\section{Author Contributions}

All authors made a significant contribution to the work reported, whether that is in the conception, study design, execution, acquisition of data, analysis and interpretation, or in all these areas; took part in drafting, revising or critically reviewing the article; gave final approval of the version to be published; have agreed on the journal to which the article has been submitted; and agree to be accountable for all aspects of the work.

\section{Funding}

The study was funded for medical writing by Allergan Aesthetics, an AbbVie company.

\section{Disclosure}

The authors report that they have no conflicts of interest. 


\section{References}

1. Shaping B, Reduction C. Body Shaping and Cellulite Reduction: Technology Proliferation Driven by Demand. Medical Insight Inc; 2009.

2. Paul M, Mulholland RS. A new approach for adipose tissue treatment and body contouring using radiofrequency-assisted liposuction. Aesthetic Plast Surg. 2009;33:687-694. doi:10.1007/s00266-0099342-z

3. Goldman A. Submental Nd: Yag laser-assisted liposuction. Lasers Surg Med. 2006;38:181-184. doi:10.1002/1sm.20270

4. Tülin Güleç A. Treatment of cellulite with LPG endermologie. Int $J$ Dermatol. 2009;48:265-270. doi:10.1111/j.1365-4632.20 09.03898.x

5. Nelson AA, Wasserman D, Avram MM. Cryolipolysis for reduction of excess adipose tissue. Semin Cutaneous Med Surg. 2009;28:244-249. doi:10.1016/j.sder.2009.11.004

6. Murray EG, Rivas OE, Stecco KA, Desilets CS, Kunz L. The use and mechanism of action of high intensity focused ultrasound for adipose tissue removal and non-invasive body sculpting: P80. Plast Reconstruct Surg. 2005;116:222-223. doi:10.1097/00006534200509011-00179

7. Coleman SR, Sachdeva K, Egbert BM, Preciado J, Allison J. Clinical efficacy of noninvasive cryolipolysis and its effects on peripheral nerves. Aesthetic Plast Surg. 2009;33:482-488. doi:10.1007/s00266008-9286-8

8. Bernstein EF. Longitudinal evaluation of cryolipolysis efficacy: two case studies. J Cosmet Dermatol. 2013;12:149-152. doi:10.1111/ jocd.12036

9. Kennedy J, Verne S, Griffith R, Falto-Aizpurua L, Nouri K. Noninvasive subcutaneous fat reduction: a review. $J$ Eur Acad Dermatol Venereol. 2015;29(9):1679-1688. doi:10.1111/jdv.12994

10. Stevens WG, Pietrzak LK, Spring MA. Broad overview of a clinical and commercial experience with Cool Sculpting. Aesthet Surg J. 2013;33(6):835-846. doi:10.1177/1090820x13494757
11. Zelickson B, Egbert BM, Preciado J, et al. Cryolipolysis for noninvasive fat cell destruction: initial results from a pig model. Dermatol Surg. 2009;35:1462-1470. doi:10.1111/j.15244725.2009.01259.x

12. Klein KB, Zelickson B, Riopelle JG, et al. Non-invasive cryolipolysis $^{\mathrm{TM}}$ for subcutaneous fat reduction does not affect serum lipid levels or liver function tests. Lasers Surg Med. 2009;41:785-790. doi:10.1002/lsm.20850

13. Raphael BA, Wasserman DI. Getting to the bare bones: a comprehensive update of non-invasive treatments for body sculpting. Curr Dermatol Rep. 2013;2(2):144-149. doi:10.1007/ s13671-013-0045-y

14. Krueger N, Mai SV, Luebberding S, Sadick NS. Cryolipolysis for noninvasive body contouring: clinical efficacy and patient satisfaction. Clin Cosmet Investig Dermatol. 2014;7:201. doi:10.2147/ccid.s44371

15. Manstein D, Laubach H, Watanabe K, Farinelli W, Zurakowski D, Anderson RR. Selective cryolysis: a novel method of non-invasive fat removal. Lasers Surg Med. 2008;40:595-604. doi:10.1002/1sm.20719

16. Avram MM, Harry RS. Cryolipolysis ${ }^{\mathrm{TM}}$ for subcutaneous fat layer reduction. Lasers Surg Med. 2009;41:703-708. doi:10.1002/ $1 \mathrm{sm} .20864$

17. Dierickx CC, Mazer JM, Sand M, Koenig S, Arigon V. Safety, tolerance, and patient satisfaction with noninvasive cryolipolysis. Dermatol Surg. 2013;39(8):1209-1216. doi:10.1111/dsu.12238

18. Ferraro GA, De Francesco F, Cataldo C, Rossano F, Nicoletti G, D'andrea F. Synergistic effects of cryolipolysis and shock waves for noninvasive body contouring. Aesthetic Plast Surg. 2012;36 (3):666-679. doi:10.1007/s00266-011-9832-7

19. Bolton J, Wu D, Goldman M. Study of a suctionless novel applicator for the treatment of pseudogynecomastia with cryolipolysis:\# E53. Lasers Surg Med. 2016;48(4).

20. Dhami LD. Liposuction. Indian J Plast Surg. 2008;41:S27. doi:10.1055/s-0039-1700473
Clinical, Cosmetic and Investigational Dermatology

\section{Publish your work in this journal}

Clinical, Cosmetic and Investigational Dermatology is an international, peer-reviewed, open access, online journal that focuses on the latest clinical and experimental research in all aspects of skin disease and cosmetic interventions. This journal is indexed on CAS.

\section{Dovepress}

The manuscript management system is completely online and includes a very quick and fair peer-review system, which is all easy to use. Visit http://www.dovepress.com/testimonials.php to read real quotes from published authors. 